\title{
Physician-Pharmacist collaboration in a pay for performance healthcare environment
}

\author{
Farley $\mathrm{TM}^{1,2}$, Izakovic $\mathrm{M}^{1,3}$ \\ Mercy Hospital, Iowa City, Iowa, USA. martin.izakovic@mercyic.org
}

\begin{abstract}
Healthcare is becoming more complex and costly in both European (Slovak) and American models. Healthcare in the United States (U.S.) is undergoing a particularly dramatic change. Physician and hospital reimbursement are becoming less procedure focused and increasingly outcome focused. Efforts at Mercy Hospital have shown promise in terms of collaborative team based care improving performance on glucose control outcome metrics, linked to reimbursement. Our performance on the Centers for Medicare \& Medicaid Services (CMS) post-operative glucose control metric for cardiac surgery patients increased from a $63.6 \%$ pass rate to a $95.1 \%$ pass rate after implementing interventions involving physician-pharmacist team based care. Having a multidisciplinary team that is able to adapt quickly to changing expectations in the healthcare environment has aided our institution. As healthcare becomes increasingly saturated with technology, data and quality metrics, collaborative efforts resulting in increased quality and physician efficiency are desirable.

CONCLUSION:Multidisciplinary collaboration (including physician-pharmacist collaboration) appears to be a viable route to improved performance in an outcome based healthcare system(Fig. 2, Ref. 12). Text in PDF www.elis.sk. KEY WORDS:physician, pharmacist, collaboration, pay for performance, clinical metrics, quality improvement.
\end{abstract}

\section{Introduction}

Collaborative care offers many potential advantages over traditional care models. Physicians are able to optimize care delivery and efficiency by delegating certain aspects of care to other members of the health care team. Care team members can include nurses, physician assistants, physical and occupational therapists, dieticians, social workers, nurse practitioners, discharge planners and clinical pharmacists. Physician-pharmacist collaboration has been increasing in both the inpatient and outpatient arenas in the United States (U.S.). Pharmacists who pursue additional residency training after earning the Doctor of Pharmacy degree (Pharm.D.) and/or are Board Certified Pharmacotherapy Specialists (B.C.P.S.) are particularly well suited for collaborative care efforts. Through collaboration with physician team leaders, pharmacists are empowered to use their specific drug knowledge and training in a way that is more fulfilling and has been shown to improve aspects of care such as blood pressure control, hemoglobin A1c, and cholesterol levels (1). Involving clinical pharmacists in inpatient team based care results in decreased length of stay, reduced adverse drug events and reduced medications errors (2).

${ }^{1}$ Mercy Hospital, Iowa City, Iowa, USA, ${ }^{2}$ University of Iowa College of Pharmacy, Iowa City, Iowa, USA, and ${ }^{3}$ St. Elizabeth University of Health and Social Sciences, Bratislava, Slovakia

Address for correspondence: M. Izakovic, MD, PhD, 500 East Market Street, Iowa City, Iowa 52245, USA.

Phone: +319.339.3622, Fax: +319.339.3788
Health care reform in the U.S. has intensified interest in pursuing and evaluating collaborative models of care. The Affordable Care Act of 2010 changed the way the physicians and hospitals were compensated in the U.S., adding compensation measures linked to quality of care $(3,4)$. The percentage of reimbursement that is tied to quality of care measures is set to increase in coming years from $1-3 \%$ initially to $20 \%$ by the year 2020 (5). The Centers for Medicare \& Medicaid Services (CMS) develops quality measures that quantify healthcare processes, outcomes, patient perceptions, and organizational structure that are associated with the ability to provide high-quality health care.

Hospitalist physicians, specialists in inpatient internal medicine, have become the cornerstone of hospital medicine in the American health system (6). They often act as organizational gatekeepers and are well positioned for improving hospital performance on quality metrics. Previously we have reported the successful integration of a clinical pharmacist into the Hospitalist Team at Mercy Hospital Iowa City (7). Duties of the clinical pharmacist include: attending rounds and interdisciplinary meetings, reviewing medication orders and conferring recommendations for drug therapy improvement, reconciling medications, providing patient education and counseling, serving as a drug information resource, pharmacokinetic drug monitoring and providing renal dose adjustments through a collaborative practice agreement. Through this collaborative relationship our quality metrics have been improved, notably in the area of glycemic control in cardiac surgery patients whose blood glucose is managed by the Hospitalist Team at Mercy.

Initiated in by the CMS and the Centers for Disease Control (CDC), the Surgical Care Improvement Project (SCIP) is 
Mercy Hospital Hospitalist Cardiac Surgery Glucose Protocol (2014):

1) Note $18-24$ hour monitoring window $=$

- $(1330$ anesthesia stop time $=0730-1330$ window next day $=$ POD \#1)

2) Keep IV insulin drip active until post operation day (POD) \#2 in AM.

- $\sim 40$ hours post operation

3) Order call to physician if blood glucose level (BGL) is $>160$ to facilitate corrective action AND to call if BGL check will fall within 90 minutes after a meal. (So BGL can be rescheduled, if reasonable)

4) Keep Diet Order Active - No Added Salt, Low Cholesterol 1800 Cal ADA Diet - starting AM POD \#1

5) Order Mealtime SQ Rapid Acting Insulin (Humalog/Novolog) supplement given IN ADDITION TO continuing insulin drip per protocol.

- To be given once patient has finished eating (TID after meals) for post op day \#1.

\begin{tabular}{lccc}
\hline $\begin{array}{l}\text { Current Infusion Rate } \\
\text { (Used to determine mealtime doses) }\end{array}$ & $\begin{array}{c}\text { SQ mealtime dose if patient eats } \\
\text { more than 50\% of meal }\end{array}$ & $\begin{array}{c}\text { SQ mealtime dose if patient eats } \\
50-25 \% \text { of meal }\end{array}$ & $\begin{array}{c}\text { SQ mealtime dose if patient eats } \\
\text { less than 25\% of meal }\end{array}$ \\
\hline 0 to 1.9 Units / Hour & 4 Units & 2 Units & 1 Unit \\
2 to 3.9 Units / Hour & 6 Units & 3 Units & 2 Units \\
4 t 5.9 Units / Hour & 8 Units & 4 Units & 3 Units \\
6 t 7.9 Units / Hour & 10 Units & 5 Units & 4 Units \\
8 to 10 Units / Hour & 12 Units & 6 Units & 5 Units \\
Over 10 Units / Hour & 14 Units & 7 Units & 6 Units \\
\hline
\end{tabular}

6) Transfer to telemetry prior to 24-hours after surgery maintaining insulin drip

7) Use IV to SQ transition protocol in AM on POD \#2 If glucose level is above $180 \mathrm{mg} / \mathrm{dl}$ is found in 18-24 hour window (1 allowed, must show correction per SCIP measure):

1) Request RN not check follow up level until 1+ hours after IV insulin bolus to allow for effect to take place

a. Make sure follow-up BGL level is done in window

2) Bolus regular insulin IV dosed at $\sim 0.1$ units $/ \mathrm{kg} \times 1$ while keeping drip going and adjusting up, per protocol.

a. Note: IV regular insulin works faster (instant peak) and clears faster ( $\mathrm{t}_{1 / 2}$ after IV insulin $\mathrm{R}$ is $30-60$ minutes) than SQ rapid acting insulin analogs (which peak in $\sim 1$ hour).

b. Can be given through our pumps (verified).

Fig. 1.The Hospitalist Team glucose management protocol for cardiac surgery patients.

aimed at improving the quality of care for surgical patients by decreasing the incidence of several postoperative complications, including surgical site infections. The SCIP-Inf-4 clinical indicator, focused at reducing deep sternal wound infections, initially measured the percentage of cardiac surgery patients with blood glucose levels less than $11.1 \mathrm{mmol} / \mathrm{L}(200 \mathrm{mg} / \mathrm{dL})$ at $6 \mathrm{AM}$ on postoperative days 1 and 2.As of January 1, 2014, The SCIPInf-4 measure was revised to evaluate postoperative blood glucose (target of $<10 \mathrm{mmol} / \mathrm{L}, \leq 180 \mathrm{mg} / \mathrm{dL}$ ) in a six-hour window on postoperative day number one (18 to 24 hours after anesthesia end time). In patients undergoing cardiac surgery, hyperglycemia on the first and second postoperative day is the single most important predictor of serious infection and complications (8). Appropriate inpatient management of hyperglycemia has been shown to improve clinical outcomes $(9,10)$. Besides aiming to affect patient outcomes, CMS will reduce payments to hospitals that fail to perform on the glucose quality measure via their hospital value-based purchasing (HVBP) initiative (11). The HVBP program, which began in 2013, attempts to improve the value of care provided to Medicare beneficiaries. The triple aim of this program is to improve healthcare quality and patient experience, while reducing healthcare costs. The HVBP composite score includes four items: patient outcome, patient experience of care, efficiency of care, and clinical process of care. The HVBP composite score provides for payment incentives and payment penalties for high- and low-performing hospitals, respectively. Cardiovascular outcomes, including cardiac surgery patient postoperative glucose control are a current HVBP outcome targets (12).

Hospitalist co-management of surgical patients adds benefits including increased rates of guideline concurrent therapy, reduced time to surgery, decreased length of stay, and fewer postoperative complications. At our institution medical co-management of diabetic patients undergoing open-heart surgery by hospitalist physicians is common practice with the primary goals of preventing complications of hyperglycemia and meeting quality measure standards. Significant work has been done at Mercy Hospital in the past using physician and pharmacist members of the hospitalist team.Medical co-management begins immediately following open-heart surgery. Patients are initiated on continuous insulin infusion per a standardized protocol with a blood glucose level goal of between 4.4 and $6.6 \mathrm{mmol} / \mathrm{L}(80$ and $120 \mathrm{mg} / \mathrm{dL}$ ). With the new surgical glucose control measure update in 2014 (SCIP-Inf-4) patient meals during the post-operative period were frequently associated with a sharp rise in blood glucose levels and a failure to meet quality measures. The Hospitalist Team clinical pharmacist took the lead on developing an approach that added mealtime subcutaneous insulin given in addition to the insulin drip to help control the post-prandial spikes (Fig. 1). Additionally the hospitalist physicians, clinical pharmacist and dietician reviewed the dietary approach and removed high carbohydrate shakes that were 


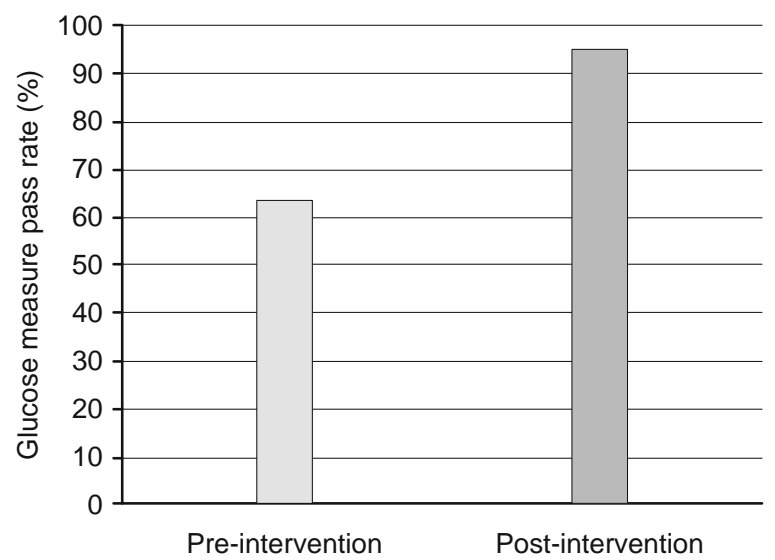

Fig. 2.Glucose quality measure pass rates for cardiac surgery patients in the care of hospitalist internal medicine physicians before (gray bar) and after (black bar) collaborative intervention.

often given post operatively. This simple, but critical intervention by the clinical pharmacist illustrates the benefit of having a multidisciplinary team to provide the key"extra set of eyes" at the right time. Since implementing these changes we have achieved at $95 \%$ success rate at meeting the quality measure in 21 patients (Fig. 2).

\section{Conclusion}

Healthcare is becoming more complex and costly in both European (Slovak) and American models. Healthcare in the U.S. (and worldwide) is undergoing change. U.S. healthcare is currently experiencing the greatest shift since the introduction of Medicare in the late1960s. Physician and hospital reimbursement are becoming less procedure focused and increasingly (clinical) outcome focused. Team based hospital care has the potential to improve outcomes. Efforts at Mercy Hospital have shown promise in terms of collaborative team based care improving performance on glucose control outcomes. Physician and pharmacist collaboration may warrant expansion as team based care attempts provide affordable, quality care.Physician-pharmacist team based care has yet to become widespread in Slovakia but may represent a worthy investment given the current focus on pay for performance financial reimbursement models. Ultimately whatever methods result in the best clinical outcomes should be considered and implemented.
Fostering worldwide communication, sharing our successes and failures, allows us to learn from each other and improve healthcare together. Having a multidisciplinary team that is able to adapt quickly to changing expectations in the healthcare environment has aided our institution. As healthcare becomes increasingly saturated with technology, data and quality metrics, collaborative efforts resulting in increased quality and physician efficiency are desirable. Multidisciplinary collaboration (including physicianpharmacist collaboration) appears to be a viable route to improved performance in an outcome based healthcare system.

\section{References}

1. Chisholm-Burns MA et al. US pharmacists' effect as team members on patient care: systematic review and meta-analyses. Med Care 2010; 48(10): 923-933.

2. Kaboli PJ et al. Clinical pharmacists and inpatient medical care: a systematic review. Arch Intern Med 2006; 166(9): 955-964.

3. Patient Protection and Affordable Care Act, in 42 U.S.C. $\$ 18001$ et seq. 2010.

4. Nelson B. Hospitalists Will Be Critical to Obamacare Success. The Hospitalist, 2014(January).

5. http://innovation.cms.gov/.

6. Wachter RM, Goldman L. The emerging role of "hospitalists" in the American health care system. N Engl J Med 1996; 335(7): 514-517.

7. Hemerson P, Izakovic M. Hospitalist-Pharmacist collaboration. Bratisl Med J 2011; 112(2): 88-91.

8. Moghissi ES. Reexamining the evidence for inpatient glucose control: new recommendations for glycemic targets. Am J Health Syst Pharm 2010; 67(16 Suppl 8): S3-8.

9. Furnary AP et al. Continuous intravenous insulin infusion reduces the incidence of deep sternal wound infection in diabetic patients after cardiac surgical procedures. Ann Thorac Surg 1999; 67(2): 352-362.

10. O'Malley CW et al. Bridge over troubled waters: safe and effective transitions of the inpatient with hyperglycemia. J Hosp Med 2008; 3(Suppl 5): 55-65.

11. National provider call: Hospital value-based purchasing. March 14, 2013, Centers for Medicare \& Medicaid Services. U.S. Government.

12. Spivack SB et al. Hospital Cardiovascular Outcome Measures in Federal Pay-for-Reporting and Pay-for-Performance Programs: A Brief Overview of Current Efforts. Circ Cardiovasc Qual Outcomes 2014; 7(5): 627-633.

Received September 30, 2014. Accepted October 21, 2014. 